\title{
Production of Staphylococcal $\alpha$-Toxin in a Defined Medium and Identification of a Stimulating Factor from Yeast Extract
}

\author{
By A. B. DALEN \\ The University of Bergen, School of Medicine, The Gade Institute, \\ Department of Microbiology, Bergen, Norway
}

(Received 22 March 1972; revised 28 July 1972)

\begin{abstract}
SUMMARY
The production of staphylococcal $\alpha$-toxin in a synthetic amino acid medium was stimulated by a factor from yeast autolysate which was identified as histidine by chromatographic methods. Addition of histidine to the medium gave an early appearance of $\alpha$-toxin, while serine and glycine stimulated its production in the late growth phase. The stimulatory capacity of serine and glycine in relation to their function as precursors in the synthesis of histidine is discussed.
\end{abstract}

\section{INTRODUCTION}

Gladstone (1938), who first obtained staphylococcal $\alpha$-toxin in a completely synthetic fluid medium, found that arginine, proline, and glycine stimulated its production. Carbon dioxide also increased the yield of toxin, though not through its effect on buffering capacity. Braman \& Norlin (I95I) reported the stimulating effect of an unidentified low molecular weight fraction from yeast; it was not thiamine or nicotinic acid, which are necessary for staphylococcal growth; it could be removed by activated carbon or dialysis. Similar effects of meat extract are possibly due to the same factor(s) (Elek, I959).

We now report the identification of the factor from yeast extract, and the effect of common amino acids on $\alpha$-toxin production in a defined medium.

\section{METHODS}

Bacterial strain. Staphylococcus aureus strain Wood 46 , phage type $42 D$ (I000 RTD), which has been cultured for several years in this laboratory, was used. It was grown on human blood agar and plated out on rabbit blood agar every two months, colonies showing wide zones of haemolysis being chosen for further propagation.

Amino acid medium. The growth requirements were studied (see Results), and the composition of the synthetic amino acid medium (Table I) based on the findings.

Incubation conditions. Bacteria were grown overnight ( 12 to $\mathrm{I} 6 \mathrm{~h}$ ) in a Casamino acid medium containing (/I): vitamin-free Casamino acids (Difco Laboratories, Detroit, Michigan, U.S.A.), $20 \mathrm{~g}$; thiamine hydrochloride, $\mathrm{O} \cdot \mathrm{I} \mathrm{mg}$; nicotinic acid, $6.0 \mathrm{mg}$; glycerol, $0.5 \%$ $(\mathrm{v} / \mathrm{v})$; and $\mathrm{K}_{2} \mathrm{HPO}_{4}, \mathrm{I} \cdot 0 \mathrm{~g}$. The $\mathrm{pH}$ was adjusted to $7 \cdot \mathrm{I}$ with $5 \mathrm{~N}-\mathrm{NaOH}$ and the medium was sterilized by Millipore filtration, pore size $0.45 \mu \mathrm{m}$. After incubation at $37^{\circ} \mathrm{C}$ on a rotary shaker, 40 to 50 strokes/min, the bacteria were centrifuged and resuspended in the amino acid medium at approximately I $\mathrm{mg}$ dry wt bacteria $/ \mathrm{ml}$ and incubated with shaking for 2 to $3 \mathrm{~h}$. Bacteria were collected by centrifuging after this pre-incubation and suspended to the desired concentration in fresh amino acid medium. 
Table I. Composition of the amino acid medium

Adjusted to $\mathrm{pH} 7 \cdot 0$ and sterilized by Millipore filtration.

L-Cystine

L-Arginine

L-Proline, L-phenylalanine

L-Glutamic acid, L-alanine, L-leucine, L-valine, L-glycine, L-lysine

L-Aspartic acid

$\mathrm{CaCl}_{2} \cdot 2 \mathrm{H}_{2} \mathrm{O}$

$\mathrm{Fe}\left(\mathrm{CH}_{3} \mathrm{COO}\right)_{3} .5 \mathrm{H}_{2} \mathrm{O}$

$\mathrm{MgSO}_{4} \cdot 7 \mathrm{H}_{2} \mathrm{O}$

$\mathrm{NaCl}$

Glucose

Sörensen phosphate buffer

Thiamine

Nicotinic acid

Pyridoxal

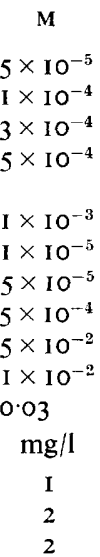

Shaking improved the peak yield of haemolysin in the amino acid medium but a gradual loss of activity was noted with continued shaking. Since the factors studied in this paper affected haemolysin production at different phases of growth, two types of incubation were employed. That with shaking was performed in $50 \mathrm{ml}$ Erlenmeyer flasks on a rotary shaker (40 strokes/min), with volumes from 3 to $5 \mathrm{ml}$, the inoculum being usually $150 \mu \mathrm{g}$ dry wt bacteria/ml. Stagnant cultures were set up in U.G.B. screw-cap flasks (United Glass Co., London) of the type usually employed for blood cultures; Io $\mathrm{ml}$ of medium gave a layer about $3 \mathrm{~mm}$ deep. The inoculum was $25 \mu \mathrm{g}$ dry wt bacteria/ml, and incubation was at $37^{\circ} \mathrm{C}$ for $24 \mathrm{~h}$.

Measurement of bacterial cell mass from fluid cultures. Suspensions were made in distilled water to give an extinction of $0 . \mathrm{I}$ to 0.4 at $700 \mathrm{~nm}$ and the dry weight was determined from a calibration curve relating dry weight to extinction.

Measurement of haemolytic activity. Rabbit erythrocytes, collected fresh daily in Alsever's solution (Kabat \& Mayer, 1964), were washed twice in phosphate-buffered saline (PBS) containing sodium chloride, $0.082 \mathrm{M}$, and Sörensen phosphate buffer, $0.03 \mathrm{M}(\mathrm{pH} 7 \cdot 0)$, then suspended in PBS to a concentration of $2 \%$.

The bacterial suspensions to be tested for $\alpha$-haemolytic activity were usually not centrifuged before titration since centrifuging made no difference to the titre. Serial doubling dilutions $(0.5 \mathrm{ml})$ of toxin were made in PBS and $0.5 \mathrm{ml}$ of erythrocyte suspension was added to each tube. After mixing, tubes were incubated in a water-bath at $37^{\circ} \mathrm{C}$ for $30 \mathrm{~min}$, then were centrifuged for $3 \mathrm{~min}$ at $750 \mathrm{~g}$. From tubes showing approximately $50 \%$ haemolysis, $0.5 \mathrm{ml}$ of the supernatant was added to $3.0 \mathrm{ml}$ of $\mathrm{I} \%$ sodium carbonate.

The solutions were kept at room temperature for at least $15 \mathrm{~min}$ before reading the extinction at 54I $\mathrm{nm}$. One haemolytic unit was defined as the amount of toxin which liberates half the haemoglobin in the test erythrocyte suspension under the conditions stated. There was a linear relationship between released haemoglobin and the amount of haemolysin up to about $70 \%$ haemolysis, but with higher concentrations the curve levelled off, 4 units of haemolysin giving only $90 \%$ haemolysis.

Rabbit erythrocytes vary by a factor of 2 to 3 in their sensitivity to staphylococcal $\alpha$ haemolysin (Bernheimer \& Schwartz, 1963). Rabbits were therefore selected whose erythro- 
cytes were of about equal sensitivity. Samples to be compared were cross-checked with erythrocytes from the same rabbit.

Column chromatography. Sephadex G-25 fine (Pharmacia AB, Uppsala, Sweden) was swelled in an ammonium formate buffer $(0.05 \mathrm{M}, \mathrm{pH} 5.0)$, which was also used as eluant. A Sephadex column, $45 \times 2.5 \mathrm{~cm}$, was used with a gel volume of $175 \mathrm{ml}$. Fractions (IO ml) were collected by an LKB Fraction Collector (LKB Produkter AB, Stockholm, Sweden) and extinction at $253.7 \mathrm{~nm}$ recorded with an LKB Uvicord Ultraviolet Absorptiometer.

The anion exchanger, Dowex-I, $8 \%$ cross-linked, 200 to $400 \mathrm{mesh}$, in chloride form (Fluka AG, Buchs, Switzerland), was converted to its formate form by suspension in $3 \mathrm{M}-$ formic acid for several days with daily changes of formic acid. Used resin was regenerated in the same manner. It was rinsed in distilled water to neutrality before use. Columns $(\mathrm{I} \cdot 5 \times$ $30 \mathrm{~cm}$ ) were used with bed volumes of $35 \mathrm{ml}$.

The cation exchanger Dowex-50 W, $8 \%$ cross-linked, I00 to 200 mesh, in H-form (Sigma Chemical Co., St Louis, Missouri, U.S.A.), was washed in $3 \mathrm{~N}-\mathrm{HCl}$ and rinsed with distilled water to neutrality before use. Resin was regenerated in the same manner. Column and bed size were as for the anion exchanger.

Thin-layer chromatography. Plastic sheets coated with cellulose (MN-polygram cel, Macherey, Nagel \& Co., Düren, Germany) were used with the following solvents (proportions by volume): (i) $n$-butanol-acetic acid-water (60:15:25); (ii) ethanol $99 \%$-M-formic acid (70:30); (iii) isopropanol-formic acid-water (40:2:10); (iv) methanol-acetone-water $(20: 4: 3)$. For identification of the yeast factor one-dimensional runs were done on $10 \times 10 \mathrm{~cm}$ plates in the different solvent systems. Samples were applied as streaks and solvents were run to the top of the plates. After drying, strips of I cm width were cut, the cellulose was scraped off, eluted in distilled water and tested for stimulating activity. Two-dimensional chromatography in appropriate solvent systems was then done, and the area containing stimulating material was removed, eluted and tested. The plates were also treated with various reagents.

Preparation of yeast extracts. A sample (I g) of yeast extract, a water-soluble autolysate in powder form (Oxoid Ltd, London, batch no. 3664550), was dissolved in $4 \mathrm{ml}$ distilled water. Ethanol (99\%) was added to $60 \%$ concentration (v/v). About $20 \%$ of the material was precipitated by the treatment and was removed by centrifugation. The supernatant was evaporated in a Rotavapor-El (Büchi, Switzerland), and the solids redissolved in $4 \mathrm{ml}$ distilled water.

Preparation of activated carbon. Activated carbon, grade KB (Atlas Chemical Industries, Wilmington, Delaware, U.S.A.) was washed in distilled water then mixed with an equal weight of Celite Hyflo-Super-Cel (Koch-Light Laboratories Ltd, Colnbrook, Buckinghamshire) to improve flow rate, and used in small columns. For step-wise elution with ethanolic solutions, volumes of $20 \mathrm{ml} / \mathrm{g}$ carbon were collected at each step.

Chemicals. Amino acids were obtained from Nutritional Biochemicals, Cleveland, Ohio, U.S.A.; vitamins were from Sigma Chemical Co.; other chemicals were of analytical grade from E. Merck, Darmstadt, Germany.

\section{RESULTS}

\section{Growth requirements of strain Wood 46 and composition of a synthetic medium}

The amino acid composition of the synthetic medium was based on the findings of Gladstone (1937) that cysteine, leucine, valine, proline, glycine, aspartic acid, phenylalanine and arginine were needed for rapid growth of staphylococci. Alanine and glutamic acid were 


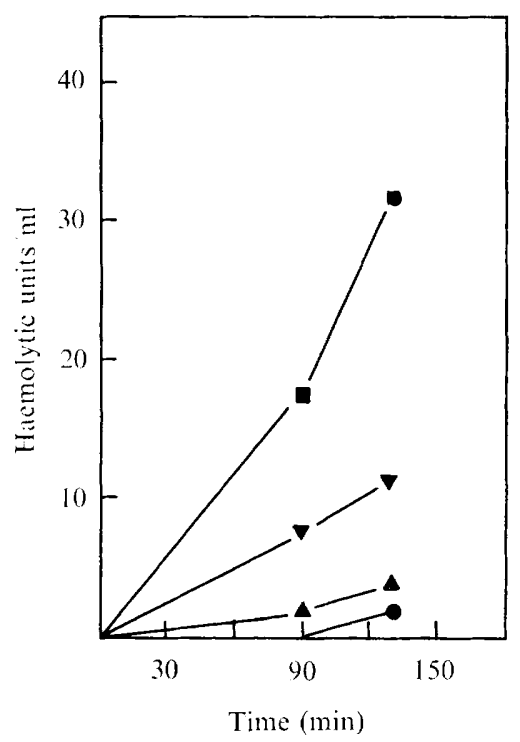

Fig. 1. Stimulating effect of a $60 \%$ ethanol extract of yeast autolysate on $\alpha$-toxin production by Staphylococcus aureus strain Wood 46 , in a defined medium in shaken culture. Inoculum, I mg dry wt bacteria $/ \mathrm{ml}$. Control: no extract added, $\bullet$; extract from I $\mu \mathrm{g}$ autolysate $/ \mathrm{ml}, \boldsymbol{\Delta} ; \mathrm{IO} \mu \mathrm{g} / \mathrm{ml}, \boldsymbol{\nabla}$; $25 \mu \mathrm{g} / \mathrm{ml}$,

stated to be unnecessary for growth but were included together with lysine in our medium. Valine, proline, arginine and glycine were essential for our strain. An estimate of the turnover of the different amino acids in their L-forms was made by incubating bacteria in a mixture of the amino acids mentioned, each at I mM, for $36 \mathrm{~h}$. The supernatant, after centrifuging and filtration, was analysed by a Beckman amino acid analyser. The results indicated a low turn-over of arginine, proline and valine, while glycine and aspartic acid were completely consumed. The relative proportions of the amino acids in the medium were adjusted approximately to the turn-over values (Table I).

Several salts were included. $\mathrm{Ca}^{2+}$ and $\mathrm{Mg}^{2+}$ have been shown to promote growth (Shooter \& Wyatt, 1955), and were included although growth took place in their absence. Calcium in high concentration inhibits $\alpha$-toxin production, but not at the concentration used here. Sodium chloride at various concentrations did not affect the maximal growth rate, but did shorten the lag period when small inocula were used. Gladstone (1938) found that magnesium and iron were required for optimal production of $\alpha$-toxin, and both ions were therefore included.

As shown for other staphylococcal strains (Knight, 1937), thiamine and nicotinic acid were essential for the growth of our strain, both in the amino acid and the Casamino acid medium. Pyridoxal showed some growth-promoting effect, but $p$-aminobenzoic acid and biotin did not.

\section{Isolation and identification of the yeast factor}

In the first investigations $\alpha$-haemolysin production was measured in shaken cultures in the amino acid medium over a 2 -h period, after various additions. The yeast autolysate itself inhibited $\alpha$-toxin activity and its effect on formation of the toxin could not therefore be evaluated. However, the interfering component was precipitated by $60 \%$ ethanol, the evaporated supernatant having the stimulating effect shown in Fig. I. Ethanol $(99 \%)$ 
extracted only traces of the factor from yeast extract powder, but methanol removed a considerable amount. Acetone precipitated it from alcoholic solutions, thus further substantiating its polar character.

A $60 \%$ ethanol extract from yeast autolysate was evaporated and the residue redissolved in distilled water. Activated carbon removed the stimulating activity, which could be eluted from the carbon by ethanol. Step-wise elution with increasing concentrations of ethanol, viz. 5,15 and $50 \%$, and $50 \%$ containing $0.05 \mathrm{~N}-\mathrm{NH}_{3}$, yielded some activity in all fractions but the highest activity was found in the $15 \%$ ethanolic and in the ammoniacal $50 \%$ ethanol fractions. This might indicate multiple stimulating substances or a varying degree of binding of a single substance to carbon.

When the four fractions were passed through Sephadex G-25 columns, the stimulating material was in each case recovered in a single fraction, with a $K_{d}$ of 0.9 ; crude $60 \%$ ethanol extract from yeast behaved identically, and was thereby considerably purified.

The activity in Sephadex-purified material was not retained by a Dowex-I anion exchange column equilibrated with formate buffer $0.05 \mathrm{M}, \mathrm{pH} 5 \cdot 0$, though it was by Dowex-50 in $\mathrm{H}^{+}$ form. Step-wise treatment with increasing concentrations of ammonium formate resulted in elution at $0.2 \mathrm{M}$.

One-dimensional thin-layer chromatography of Sephadex-purified material on cellulose was done in different solvent systems, and activity was confined to one or two of the adjacent zones of I cm width. The $R_{F}$ values from such runs served to locate the active area in twodimensional runs. In these, three spots were revealed by exposure to iodine vapour and two by silver nitrate. All five spots lay, however, outside the active area. When treated with a ninhydrin-collidine reagent (Randerath, I966), nine spots appeared. One, which had a greyish colour, corresponded to the active area. The Pauly reagent stained it red, indicating an imidazole group. When histidine was chromatographed in the same solvent systems, it gave the same $R_{F}$ values as the stimulating substance.

When histidine at I mM was added to the amino acid medium inoculated with I $\mathrm{mg}$ dry weight of bacteria/ml, production of $\alpha$-toxin started within I $h$, as with the yeast factor.

One-dimensional chromatography of the ethanol yeast extract gave several positive spots with the Pauly reagent. One major spot had an $R_{F}$ value equal to that of urocanic acid, a second corresponded to histidine and a third remained at the starting point, presumably representing histidine peptides.

\section{Effect of amino acids on $\alpha$-toxin production}

Shaken cultures with inocula of $150 \mu \mathrm{g}$ dry wt bacteria/ml were used to detect early stimulating activity, and stagnant cultures with inocula of $25 \mu \mathrm{g} / \mathrm{ml}$ incubated for $24 \mathrm{~h}$ for delayed stimulation. Leucine, isoleucine, lysine, methionine, aspartic acid, asparagine, proline, hydroxyproline, threonine, glutamic acid, glutamine, tyrosine, tryptophan, alanine, valine and phenylalanine had no effect on toxin production in concentrations of from $\mathrm{I}$ to Io mM. Arginine, glycine, serine and histidine increased the yield of $\alpha$-toxin.

Arginine, as noted above, was required for growth. No haemolysin was formed in nongrowing arginine-deprived cultures. When the arginine concentration was increased from $0 . \mathrm{I} \mathrm{mM}$ to $\mathrm{I} \mathrm{mM}$ in the amino acid medium, the haemolytic titre was about $50 \%$ higher than in the stationary control cultures after $24 \mathrm{~h}$, but the stimulating activity of arginine was low compared with that of histidine, serine or glycine. The effect of glycine resembled that of serine, but was smaller. The stimulating effects of histidine and serine showed striking differences. In the control cultures haemolysin appeared in the late phase of growth, the length of the latent period being inversely related to the size of inoculum. With serine the 


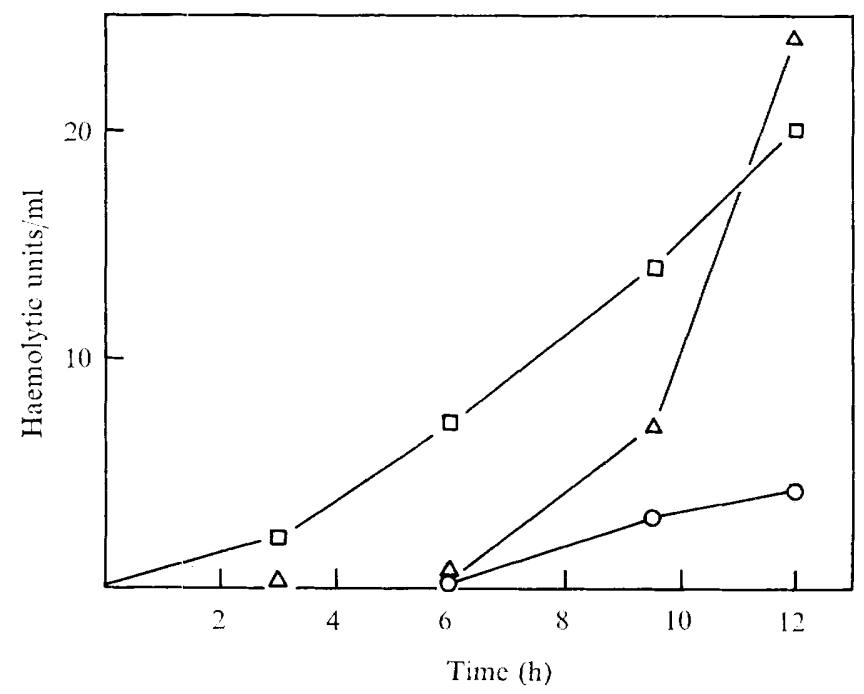

Fig. 2. Stimulating effects of serine and histidine on $\alpha$-toxin production in stagnant cultures of Staphylococcus aureus strain Wood 46 . Inoculum, $25 \mu \mathrm{g}$ dry wt bacterial $/ \mathrm{ml}$. Amino acid medium, $\mathrm{O}$; amino acid medium with histidine (I mM), [] ; amino acid medium with serine (I $\mathrm{mM}$ ) $\triangle$.

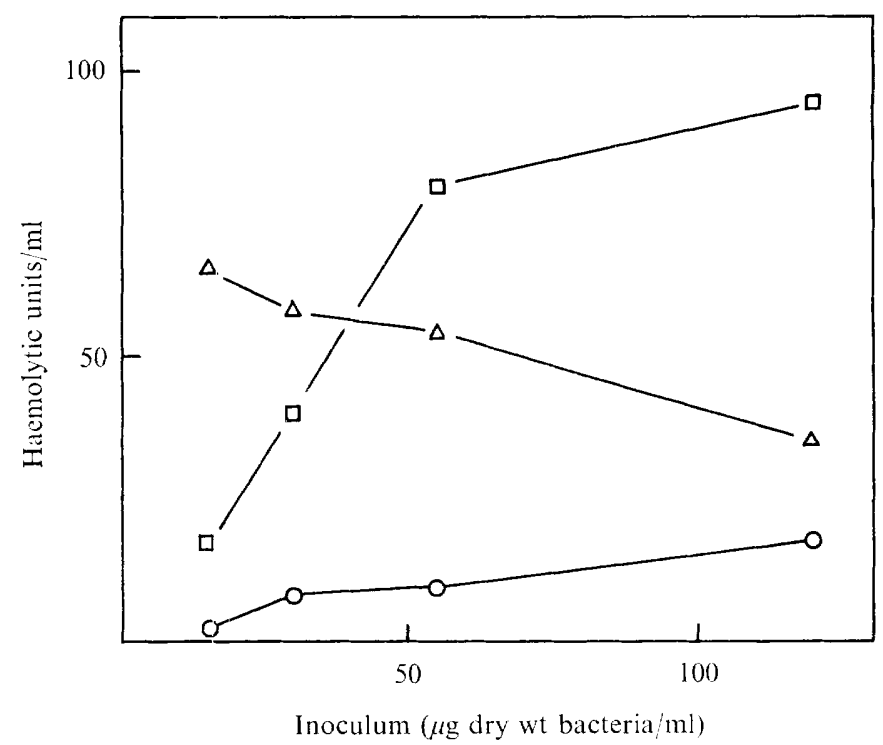

Fig. 3. Influence of the size of the inoculum on the stimulating activities of serine and histidine in stagnant cultures of Staphylococcus aureus strain Wood 46. Incubation, $24 \mathrm{~h}$. Amino acid medium, $\bigcirc$; amino acid medium with serine (I mM), $\triangle$; amino acid medium with histidine ( $\mathrm{mm}$ ), $\square$.

latent period was the same but yield of haemolysin was increased (Fig. 2). Histidine, however, shortened the latent period, haemolysin appearing in the early growth phase.

In shaken cultures with large inocula histidine stimulated production of haemolysin within minutes. Shaking and small volumes, which ensured good aeration, generally enhanced the effect. Serine stimulation required long incubation, and shaking lowered the haemolytic activity, presumably by inactivating $\alpha$-toxin. 


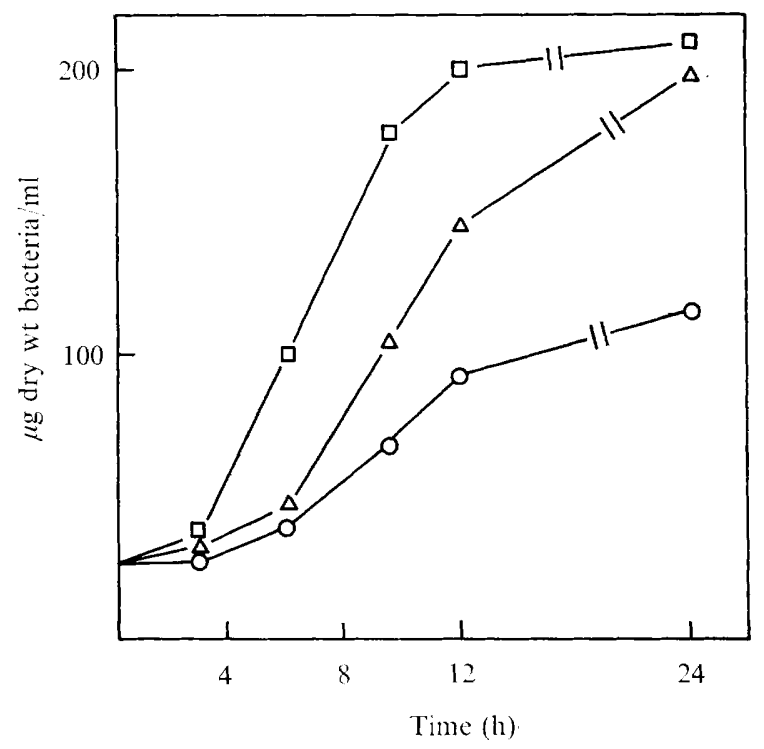

Fig. 4. Effects of serine and histidine on growth in stagnant cultures of Staphylococcus aureus strain Wood 46. Amino acid medium, $O$; amino acid medium with serine (I $\mathrm{mM}$ ), $\triangle$; amino acid medium with histidine (I mM), $\square$.

In stagnant cultures the size of the inoculum had a pronounced effect (Fig. 3). Thus the stimulating effect of serine was inversely, and that of histidine directly, related to inoculum size.

Both serine and histidine affected the growth rate of bacteria in the amino acid medium. With low inocula (below I $\mu \mathrm{g}$ dry $\mathrm{wt} / \mathrm{ml}$ ) growth was apparent within $\mathrm{I} 2 \mathrm{~h}$ in histidine cultures, but more than $24 \mathrm{~h}$ elapsed before growth occurred in the controls and the serine cultures. In Fig. 4 a growth curve from a stagnant culture is shown. Doubling time in the maximal growth phase was $140 \mathrm{~min}$ with histidine, $190 \mathrm{~min}$ with serine and $290 \mathrm{~min}$ in the control culture. Shaking shortened doubling times by about $30 \mathrm{~min}$.

\section{DISCUSSION}

The sudden appearance of $\alpha$-haemolysin both intra- and extracellularly during certain phases of growth suggests that it is an induced protein (Hendricks \& Altenbern, 1968). The amino acid medium used in this study was designed to give suitable conditions for detecting an inducing substance. Gladstone (1938) followed haemolytic activity for from I to 15 days after inoculation and his results are not directly comparable with mine. Thus, under my conditions, proline had no stimulating effect, while the stimulating effect of glycine and to a certain extent arginine was confirmed. Serine was apparently not studied by Gladstone, since it inhibited the growth of staphylococci (Fildes, Richardson, Knight \& Gladstone, 1936). He found histidine to be without effect, probably because of the small inocula and the prolonged incubation.

As far as it was characterized, the unidentified stimulating yeast factor of Braman \& Norlin (I95I) behaved in the same way as histidine. Yeast extract is rich in histidine and histidine peptides. Because of the potent proteolytic activity of staphylococci, one would also expect these peptides to stimulate haemolysin production.

The rapid response to histidine suggests that it is an inducer of $\alpha$-toxin. 
The stimulating effect of $\mathrm{CO}_{2}$ (Kapral, 1966), serine and glycine may depend on the fact that Staphylococcus aureus has a biosynthetic pathway for histidine similar to that in Salmonella (Kloos \& Pattee, I965). Histidine is formed from ATP in a complex sequence involving ten steps and nine enzymes. Glycine and $\mathrm{CO}_{2}$ are precursors in the de novo synthesis of purines (Buchanan, Sonne \& Deluva, 1948), and serine may contribute to the purine molecule by being degraded to glycine. The three compounds are therefore precursors of histidine, and their stimulating effect on $\alpha$-toxin production might be related to this fact. The delayed effect of serine and glycine might be due to more of the purine pool being available for histidine synthesis in the late exponential phase.

The possibility exists that $\alpha$-toxin production is related in a more direct manner to the size of the purine pool. Serine and glycine as precursors might affect it and histidine might have a sparing effect on it due to repression of the histidine operon (Ames \& Garry, 1959). Since histidine has a pronounced growth-enhancing effect, induction of $\alpha$-toxin would be under 'non-gratuitous' conditions. Further experiments are therefore required before any definite conclusions on the inductive properties of histidine can be made.

\section{REFERENCES}

AMEs, B. N. \& GARry, B. (I959). Coordinate repression of the synthesis of four histidine biosynthetic enzymes by histidine. Proceedings of the National Academy of Sciences of the United States of America 45, 453.

Bernheimer, A. W. \& SChwartz, L. (1963). Isolation and composition of staphylococcal $\alpha$-toxin. Journal of General Microbiology 30, 455-468.

Braman, J. \& Norlin, G. (I95I). A factor in yeast dialysate of significance in the formation of staphylococcus toxin. Acta pathologica et microbiologica scandinavica 29, I 27-I3I.

Buchanan, J. M., Sonne, J. C. \& Deluva, A. M. (I948). Biological precursors of uric acid; role of lactate, glycine, and carbon dioxide as precursors of carbon chain and nitrogen atom 7 of uric acid. Journal of Biological Chemistry $\mathbf{1 7 3}, 8 \mathrm{I}-98$.

EleK, S. D. (1959). Staphylococcus pyogenes and Its Relation to Disease, p. 223. Edinburgh and London: E. and S. Livingstone.

Fildes, P., Richardson, G. M., Knight, B. C. J. G. \& Gladstone, G. P. (i936). A nutrient mixture suitable for the growth of Staphylococcus aureus. British Journal of Experimental Pathology I7, 48 I-484.

Gladstone, G. P. (1937). The nutrition of Staphylococcus aureus: nitrogen requirements. British Journal of Experimental Pathology 18, 322-333.

Gladstone, G. P. (1938). The production of staphylococcal $\alpha$-haemolysin in a chemically-defined medium. British Journal of Experimental Pathology 19, 208-226.

Hendricks, C. W. \& Altenbern, R. A. (1968). Studies on the synthesis of staphylococcal alpha toxin. Canadian Journal of Microbiology 14, 1277-1281.

KABAt, E. A. \& MAYer, M. M. (1964). Experimental Immunochemistry, p. 149. Springfield, Illinois: Charles C. Thomas.

Kapral, F. A. (1966). Studies on the induction of Staph. aureus alpha-toxin. Bacteriological Proceedings M35, 44 .

Kloos, W. E. \& PAtTeE, P. A. (1965). A biochemical characterization of histidine-dependent mutants of Staphylococcus aureus. Journal of General Microbiology 39, I85-194.

KNIGHT, B. C. J. G. (1937). The nutrition of Staphylococcus aureus: nicotinic acid and vitamin $B_{1}$. Biochemical Journal 3I, 73 I -737 .

Randerath, K. (1966). Thin-layer Chromatography, p. I I5. New York and London: Academic Press.

Shooter, R. A. \& WYATT, H. V. (I955). Mineral requirements for growth of Staphylococcus pyogenes. Effect of calcium and magnesium ions. British Journal of Experimental Pathology 36, 34I-350. 